CAÑIZARES, K.A.L.; GOTO, R. Crescimento e produção de híbridos de pepino em função da enxertia. Horticultura Brasileira, Brasília, v. 16, n. 2, p. 110-13, 1998.

CAÑIZARES, K.A.L.; IOZI, R.N.; STRIPARI, P.C.; GOTO, R. Enxertado, japonês fica mais brilhante. Agrianual 97. Anuário Estatístico da Agricultura Brasileira. São Paulo, FNP, Consultoria \& Comércio, 1996. p. 332-333.

FRIEDLANDER, D., ATSMON, D., GALUN, E. The effect of grafting on sex expression in cucumber. Plant Soil, v. 18, p. 1343-1350, 1977. FUJIEDA, K. Cucumber. In: KONISHI, K.; IWAHORI, S.; KITAGAWA, H.; YAKUWA, T. Horticulture in Japan. Tokio, Asakura Publishing Co., Ltda., 1986. 180 p.

JANICK, J. A ciência da horticultura. Rio de Janeiro: Freitas Bastos, 1966. p. 224-329.

JANOWSKI, G.; SKAPSKI, H. Hydro-peat method for greenhouse cucumber production. Acta Horticulturae, Wageningen, v. 156, p. 27-33, 1985.
KAWAIDE, T. Utilization of rootstocks in cucurbits production in Japan. Japanese Agricultural Research Quaterly, Tokio, v. 18, n. 4, p. 285-288, 1985

MIGUEL, A. Injerto en hortalizas. Generalitat Valenciana, Conselleria de Agricultura, pesca y alimentación, 1997. 88 p.

ODA, M. New grafting methods for fruit-bearing vegetables in Japan. Japanese Agricultural Research Quaterly, Tokio, v. 29, p. 187-194, 1995. ODA, M.; TSUJI, K.; SASAKI, H. Effect of hypocotyl morphology on survival rate and grow of cucumber seedlings grafted on Cucurbita spp. Japanese Agricultural Research Quaterly, Tokio, v. 26, p. 259-263, 1993.

RACHOW-BRANDT, G.; KOLLMANN, R. Studies on graft unions. IV. Assimilate transport and sieve element restitution in homo and heterografts. Journal Plant Physiology, v. 139, p. 584-589, 1992a.
RACHOW-BRANDT, G.; KOLLMANN, R. Studies on graft unions. V. Unloading of assimilates in homo and heterografts. Journal. Plant Physiology, v. 139, p. 579-583, 1992 b.

STRIPARI, P.C.; IOZI, R.N.; CAÑIZARES, K.A.L.; TRAVASSOS, L.H.; GOTO, R. Influência do intervalo de semeadura na sobrevivência e na qualidade de mudas de pepino enxertado em abóbora. Actas de Horticultura, v. 16, p. 33-37, 1997.

TAKAHASHI, H.; SAITO, T.; SUGE, H. Intergeneric translocation of floral stimulus a graft in monoecious cucurbitaceae with special reference to sex expression of flowers. Plant Soil, v. 23, n. 1, p. 1-9, 1982.

YAMAKAWA, K. Use of rootstocks in Solanaceous fruit-vegetable production in Japan. Japanese Agricultural Research Quaterly, v. 15 , n. 3, p. 175-179, 1982.

MELLO, S.C.; DECHEN, A.R.; MINAMI, K. Influência do boro no desenvolvimento e na composição mineral do pimentão. Horticultura Brasileira, Brasília, v. 20, n. 1, p. 99-102, março 2.002.

\title{
Influência do boro no desenvolvimento e na composição mineral do pimentão ${ }^{1}$.
}

\author{
Simone C. Mello²; Antonio Roque Dechen ${ }^{3}$; Keigo Minami ${ }^{3}$ \\ ${ }^{2}$ Rua das Magnólias, 135, Jd. Novo Mundo, 13.211-610 Jundiaí, SP; ${ }^{3}$ ESALQ, C. Postal 09, 13.418-900 Piracicaba-SP. E-mail: \\ secmello@zaz.com.br
}

\section{RESUMO}

O experimento foi conduzido no município de Piracicaba (SP), de fevereiro a junho de 1994, em solo classificado como Terra Roxa Estruturada eutrófica A moderado textura argilosa. Visou-se estudar a aplicação de boro no desenvolvimento e na composição mineral de plantas de pimentão cv. Magda. Adotou-se o delineamento em blocos casualizados com quatro doses de bórax aplicadas nas covas de plantio $\left(0 ; 5 ; 10\right.$ e $20 \mathrm{~kg} \mathrm{ha}^{-1}$ equivalentes a $0 ; 0,6 ; 1,1$ e $2,2 \mathrm{~kg} \mathrm{ha}$ ${ }^{1}$ de B) e quatro repetições. Avaliou-se a altura das plantas, o número de folhas e de flores, a área foliar, o peso da matéria seca da parte aérea e os teores de nutrientes nas folhas recém-maduras mais pecíolos, aos 45 dias após o transplante. Foram avaliados também, aos 40 dias após o transplante, o peso do material verde dos frutos e a composição mineral do pericarpo e das sementes dos mesmos. As doses de bórax não influenciaram as características avaliadas e os teores de macronutrientes e de $\mathrm{B}, \mathrm{Cu}, \mathrm{Fe}, \mathrm{Mn}$ e $\mathrm{Zn}$ nas folhas mais pecíolos. Entre as partes dos frutos, os teores de B foram superiores nas sementes em relação ao pericarpo, nas quatro doses utilizadas.

\section{ABSTRACT}

Influence of boron application on sweet pepper development and mineral composition.

An experiment was carried out in Piracicaba (Brazil) from February to June 1994, to study the effects of boron application on the development and mineral composition of sweet pepper (Capsicum annuиm L.), cultivar Magda. The experiment was carried out in a randomized block design with four levels of borax $(0 ; 5 ; 10$ and $20 \mathrm{~kg}$ ha ${ }^{-1}$ equivalent to $0 ; 0.6 ; 1.1$ and $2.2 \mathrm{~kg} \mathrm{ha}^{-1}$ of B) and four replications. The plant height, number of leaves and flowers were evaluated, also the foliar area, dry weight of aerial part and nutrient contents in the leaves plus petioles at 45 days after transplanting date. In addition, fruit fresh weight; pericarp and seed mineral composition were evaluated 40 days after transplanting date. Different concentrations of borax did not affect the evaluated characteristics nor the macronutrient and $\mathrm{B}, \mathrm{Cu}, \mathrm{Fe}, \mathrm{Mn}$ and $\mathrm{Zn}$ levels in the leaves plus petioles and fruits. For the pericarp and seeds, the concentrations of boron were higher in the seeds than of in petioles for all levels of applied borax.

Palavras-chave: Capsicum annuum, boro, bórax, nutrição mineral.

Keywords: Capsicum annuum, boron, borax, mineral nutrition.

\section{(Aceito para publicação em 27 de novembro de 2.001)}

$\mathrm{O}$ pimentão (Capsicum annuum L.), apesar de sua reconhecida importância econômica, é pouco estudado no Brasil, no que diz respeito à adubação com micronutrientes, especialmente o boro. Além de afetar o desenvolvimento e a produção do pimentão, o boro pode interferir no equilíbrio nutricional da cultura. Segundo Correia et al. (1977), a deficiência de B em plantas de pimentão reduz o peso do material seco da parte aérea e das raízes, o nú-

\footnotetext{
${ }^{1}$ Parte da dissertação de mestrado apresentada pelo primeiro autor à ESALQ/USP.
} 
mero de ramos, de folhas e de flores. Além desses efeitos, o B também reduz a área foliar e a taxa fotossintética, conforme verificado por Pandev et al. (1982). Na nutrição mineral de plantas, o fornecimento desse micronutriente nas raízes influencia a absorção, o acúmulo e a utilização de outros elementos essenciais (Salinas et al., 1982). Santos et al. (1990) obtiveram teores foliares maiores de $\mathrm{Mg}$ e de $\mathrm{Cu}$, em plantas de pimentão, com a aplicação de $2 \mathrm{mg} \mathrm{L}^{-1}$ de $\mathrm{B}$ em solução nutritiva.

A espécie Capsicum annuum é muito sensível à deficiência de $\mathrm{B}$, sendo que a sua aplicação via solo tem sido uma prática adequada para aumentar a produtividade da cultura. Horino et al. (1980) constataram incrementos de 6,7 t ha- ${ }^{-1}$ na produção de pimentão com a aplicação de $2 \mathrm{~kg} \mathrm{ha}^{-1}$ de B. Trani \& Raij (1996) recomendam a adição de $1,0 \mathrm{~kg}$ $\mathrm{ha}^{-1}$ de B no plantio do pimentão, independentemente do teor desse elemento no solo. Entretanto, essas quantidades, normalmente empregadas, que variam de 1 a $2 \mathrm{~kg} \mathrm{ha}^{-1}$ de $\mathrm{B}$, podem não ser adequadas para o desenvolvimento da cultura, levando-se em consideração o teor de B no solo, o tipo de solo e a época de cultivo. Navrot \& Levin (1976) observaram em solo turfoso com teor médio de $0,93 \mathrm{mg} \mathrm{dm}^{-3}$ de $\mathrm{B}$ que a adição de $2 \mathrm{~kg} \mathrm{ha}^{-1}$ desse elemento diminuiu a produção de pimentão. Na cultura do tomateiro, pertencente à mesma família do pimentão, Plese et al. (1998) obtiveram redução no peso médio de frutos com a aplicação de 1 e $2 \mathrm{~g} \mathrm{cova}^{-1}$ de ácido bórico, equivalente a 3,4 e 6,8 $\mathrm{kg} \mathrm{ha}^{-1}$ de boro, em solo com 0,56 mg $\mathrm{dm}^{-3}$ de B (água quente). Gunes et al. (1999), também em plantas de tomate, observaram sintomas de toxidez de B com a adição de 10 e $20 \mathrm{mg} \mathrm{kg}^{-1}$ desse elemento no solo. Realizou-se, portanto, esse trabalho com o objetivo de verificar os efeitos da aplicação de B no desenvolvimento e na composição mineral do pimentão, cv. Magda, em solo com 1,0 $\mathrm{mg} \mathrm{dm}^{-3}$ de B.

\section{MATERIAL E MÉTODOS}

O experimento foi conduzido no município de Piracicaba (SP), no período de fevereiro a junho de 1994, em solo classificado como Terra Roxa Estruturada eutrófica A moderado textura argilosa. A área, amostrada à profundidade de $20 \mathrm{~cm}$, apresentou as seguintes características químicas: $\mathrm{pH}$ $\left(\mathrm{CaCl}_{2}\right) 5,2 ; 40,4 \mathrm{~g} \mathrm{dm}^{-3}$ de matéria orgânica; $888 \mathrm{mg} \mathrm{dm}^{-3}$ de P (resina); 9,6, 22,2 e 15,4 $\mathrm{mmol}_{\mathrm{c}} \mathrm{dm}^{-3} \mathrm{de} \mathrm{K}, \mathrm{Ca}$ e $\mathrm{Mg}$, respectivamente; acidez potencial 27,7 mmol $_{\mathrm{c}} \mathrm{dm}^{-3}$; capacidade de troca de cátions $75 \mathrm{mmol}_{\mathrm{c}} \mathrm{dm}^{-3}$; saturação por bases $63 \% ; 1,0 \mathrm{mg} \mathrm{dm}^{-3}$ de boro.

$\mathrm{O}$ delineamento experimental foi de blocos casualizados com quatro repetições, sendo os tratamentos compostos pelas doses de bórax (11,5\% B) 0; 5; 10 e $20 \mathrm{~kg} \mathrm{ha}^{-1}$, que foram equivalentes a $0 ; 0,6 ; 1,1$ e $2,2 \mathrm{~kg} \mathrm{ha}^{-1}$ de B. As aplicações do bórax foram feitas em covas de $20 \times 20 \times 20 \mathrm{~cm}$, misturando-se bem com o solo a fim de homogeneizar. Com base nos resultados da análise química do solo, dispensou-se a adubação básica de plantio. Foram aplicados, aos 10; 25; 40 e 55 dias após o transplante, 2,5 $\mathrm{g} /$ planta de $\mathrm{Ne} 1,0 \mathrm{~g} /$ planta de $\mathrm{K}_{2} \mathrm{O}$ (Passos, 1985), empregando-se como fonte a uréia $(45 \% \mathrm{~N})$ e o cloreto de potássio $\left(60 \% \mathrm{~K}_{2} \mathrm{O}\right)$.

As mudas produzidas em bandejas de isopor com 128 células, preenchidas com substrato composto de casca processada e enriquecida, vermiculita expandida e turfa processada e enriquecida, foram transplantadas em 24 de fevereiro de 1994, no espaçamento de 1,0 entre fileiras e $0,5 \mathrm{~m}$ entre plantas. Cada parcela experimental foi composta de 20 plantas, com uma densidade de 20.000 plantas/ha.

Para as avaliações da altura de plantas, número de flores, número de folhas, estimativa da área foliar, peso do material seco da parte aérea e teores de nutrientes nas folhas mais pecíolos, aos 45 dias após o transplante, amostraram-se três plantas/parcela. Para a determinação da área foliar, utilizou-se o método de uso de áreas conhecidas descrito por Benincasa (1988).

A colheita dos frutos iniciou-se em 5 de abril e se estendeu até 14 de junho de 1994. Os frutos foram pesados, para a avaliação do peso médio de frutos por planta. Para a análise química dos mesmos, coletaram-se cinco frutos ao acaso por parcela, aos 40 dias após o trans- plante, que foram separados em pericarpo (P) e sementes (S). Empregouse para determinação dos teores de nutrientes a metodologia descrita por Sarruge \& Haag (1974) e Bataglia et al. (1983): Nitrogênio: método semimicroKjeldahl; fósforo: método colorimétrico; enxofre, potássio, cálcio, magnésio, cobre, ferro, manganês e zinco: espectrofotometria de absorção atômica; B: colorimetria da azometina $\mathrm{H}$.

\section{RESULTADOS E DISCUSSÃO}

Considerando-se o desenvolvimento das plantas no campo, pôde-se observar que aquelas que receberam a aplicação de $20 \mathrm{~kg}^{-1}$ de bórax $(2,2 \mathrm{~kg}$ ha${ }^{1}$ de B) apresentaram sintomas iniciais de toxidez, aos quinze dias após o transplante. Ocorreu amarelecimento das pontas das folhas seguido por necrose. Além disso, as plantas tinham crescimento mais lento em comparação com a testemunha. Contudo, tais sintomas desapareceram ao longo do desenvolvimento da cultura. Este fato sugere que quando as plantas estavam no início do desenvolvimento a dose de boro em questão foi excessiva, levando-se em consideração o teor de boro no solo (1,0 $\mathrm{mg} \mathrm{dm}^{-3}$ ), considerado alto segundo Raij et al. (1996), bem como o modo de aplicação, ou seja, na cova de plantio, que proporcionou concentração elevada e localizada desse micronutriente, próximo às raízes, resultando, dessa forma, em sintomas de toxicidade. Salinas et al. (1982) também verificaram efeitos fitotóxicos do B em plantas de pimentão com o aumento das doses em solução nutritiva $(0 ; 0,25 ; 1 ; 2 ; 4 ; 8$ e $16 \mathrm{mg}$ $\mathrm{L}^{-1}$ de B). Resultados semelhantes foram obtidos por Gunes et al. (1999) em plantas de tomate, as quais apresentaram sintomas de toxidez com a adição de 10 e $20 \mathrm{mg} \mathrm{kg}^{-1}$ de boro no solo. Para a altura de plantas, número de folhas, número de flores, área foliar e peso do material seco da parte aérea as análises de variâncias não acusaram efeitos significativos entre as doses de boro, cujos valores constam da Tabela 1 . O peso do material verde dos frutos, cujo valor médio foi de $84 \mathrm{~g} /$ fruto, amostrados aos 40 dias após o transplante, também não foi afetado pelos tratamentos empregados. Tais resultados indicam que o teor 
Tabela 1. Valores médios de altura de plantas, número de folhas, número de flores, estimativa da área foliar e peso do material seco da parte aérea, aos 45 dias após o transplante. Piracicaba, ESALQ, 1994.

\begin{tabular}{lccccc}
\hline $\begin{array}{c}\text { Doses de bórax } \\
\text { (kg/ha) }\end{array}$ & Altura (cm) & $\begin{array}{c}\text { Número de } \\
\text { folhas }\end{array}$ & $\begin{array}{c}\text { Número de } \\
\text { flores }\end{array}$ & $\begin{array}{c}\text { Área foliar } \\
\text { (dm2) }\end{array}$ & $\begin{array}{c}\text { Peso do } \\
\text { material seco } \\
\text { (g/planta) }\end{array}$ \\
\hline 0 & 42,4 & 104,4 & 18,9 & 18,1 & 13,9 \\
5 & 42,5 & 100,7 & 19,5 & 17,4 & 13,1 \\
10 & 43,6 & 101,8 & 16,8 & 19,1 & 14,4 \\
20 & 42,8 & 135,3 & 25,8 & 20,9 & 14,9 \\
\hline C. V. (\%) & 7,33 & 14,82 & 31,27 & 14,20 & 16,13 \\
\hline
\end{tabular}

Tabela 2. Teores de macronutrientes e de micronutrientes no pericarpo (P) e nas sementes (S) de pimentão cv. Magda, aos 40 dias após o transplante. Piracicaba, ESALQ, 1994.

\begin{tabular}{|c|c|c|c|c|c|c|c|c|c|c|c|c|}
\hline \multirow{2}{*}{$\begin{array}{c}\text { Doses } \\
\text { de } \\
\text { bórax } \\
\left(\mathrm{kg} \mathrm{ha}^{-1)}\right.\end{array}$} & \multirow{2}{*}{$\begin{array}{c}\text { Partes } \\
\text { do fruto }\end{array}$} & $\mathbf{N}$ & $\mathbf{P}$ & $\mathbf{K}$ & $\mathrm{Ca}$ & Mg & $\mathbf{S}$ & B & $\mathrm{Cu}$ & $\mathrm{Fe}$ & Mn & $\mathrm{Zn}$ \\
\hline & & \multicolumn{6}{|c|}{$\mathbf{g ~ k g}^{-1}$} & \multicolumn{5}{|c|}{$\mathrm{mg} \mathrm{kg}^{-1}$} \\
\hline \multirow[t]{2}{*}{0} & $P$ & $22,3 a$ & $3,6 a$ & $28,6 a$ & $1,4 a$ & $1,6 a$ & $1,9 a$ & $19,7 a$ & $11,3 a$ & $88,0 a$ & $77,3 a$ & $33,5 a$ \\
\hline & $S$ & $29,6 a$ & $5,2 a$ & $23,4 a$ & $1,2 a$ & $3,2 a$ & $1,7 a$ & $30,1 \mathrm{~b}$ & $14,5 a$ & $92,5 a$ & $79,3 a$ & $36,8 a$ \\
\hline \multirow[t]{2}{*}{5} & $P$ & $21,7 a$ & $3,5 a$ & $31,0 a$ & $1,9 a$ & $1,8 a$ & $1,4 a$ & $14,9 a$ & $8,5 a$ & $69,5 a$ & $77,0 a$ & $28,5 a$ \\
\hline & $S$ & $28,1 a$ & $5,2 a$ & $23,8 a$ & $1,7 a$ & $3,2 a$ & $1,6 a$ & $48,5 \mathrm{~b}$ & $13,0 a$ & $87,0 a$ & $79,3 a$ & $31,0 a$ \\
\hline \multirow[t]{2}{*}{10} & $P$ & $22,2 a$ & $3,8 a$ & $31,4 a$ & $1,3 a$ & $1,9 a$ & $1,7 a$ & $16,2 a$ & $11,5 a$ & $83,5 a$ & $76,8 a$ & $32,0 a$ \\
\hline & $S$ & $28,6 a$ & $5,7 a$ & $21,4 a$ & $1,2 a$ & $2,9 a$ & $1,3 a$ & $33,9 \mathrm{~b}$ & $13,7 a$ & $89,7 a$ & $78,0 \mathrm{a}$ & $34,3 a$ \\
\hline \multirow[t]{2}{*}{20} & $P$ & $22,2 a$ & $3,7 a$ & $32,1 a$ & $2,1 a$ & $1,8 a$ & $1,3 a$ & $17,5 a$ & $9,0 a$ & $64,3 a$ & $77,5 a$ & $35,0 a$ \\
\hline & $S$ & $30,3 a$ & $4,9 a$ & $23,3 a$ & $1,3 a$ & $2,9 a$ & $1,3 a$ & $41,9 \mathrm{~b}$ & $13,8 a$ & $76,8 a$ & $78,5 a$ & $31,0 a$ \\
\hline \multirow[t]{2}{*}{ Média } & $P$ & $22,1 a$ & $3,7 a$ & $30,8 a$ & $1,7 a$ & $1,8 a$ & $1,5 a$ & $16,9 a$ & $10,1 a$ & $76,3 a$ & $77,1 \mathrm{a}$ & $32,3 a$ \\
\hline & $S$ & $29,2 \mathrm{~b}$ & $5,3 \mathrm{~b}$ & $23,0 \mathrm{~b}$ & $1,3 a$ & $3,1 \mathrm{~b}$ & $1,5 a$ & $37,7 \mathrm{~b}$ & $13,8 b$ & $86,5 \mathrm{~b}$ & 78,8 b & $33,3 \mathrm{~b}$ \\
\hline C. V. $(\%)$ & $P$ & 7,47 & 9,83 & 21,98 & 42,09 & 19,65 & 27,74 & 24,9 & 12,0 & 10,7 & 1,0 & 12,2 \\
\hline
\end{tabular}

*/ Médias seguidas pela mesma letra não diferem entre si pelo teste de Tukey, a $5 \%$ de probabilidade.

de B presente no solo foi considerado adequado para o desenvolvimento da cultura. Resultados semelhantes foram obtidos por Santos et al. (1990), os quais observaram que o número de folhas definitivas e o peso médio dos frutos não foram afetados pelas doses de $\mathrm{B}(0,25$; 0,$50 ; 1,00$ e $\left.2,00 \mathrm{mg} \mathrm{L}^{-1}\right)$. Entretanto Navrot \& Levin (1976) constataram que a aplicação de $2 \mathrm{~kg}$ ha $^{-1} \mathrm{de} \mathrm{B}$ em solo turfoso com $0,93 \mathrm{mg} \mathrm{dm}^{-3}$ de B reduziu a produção da cultura. Cabe ressaltar, também, que o fato da maior dose de B não ter reduzido as características avaliadas, confirma a recuperação das plantas, que no início apresentaram sintomas de fitotoxidez, e sugere que o pimentão cv. Magda apresenta tolerância ao excesso de B. De acordo com Adriano (1986), o pimentão pode ser considerado uma espécie semi-tolerante ao excesso desse micronutriente no substrato.
Para os teores de nutrientes nas folhas mais pecíolos a análise de variância não acusou efeito significativo entre as doses de bórax, sendo que os teores médios de $\mathrm{N}, \mathrm{P}, \mathrm{K}, \mathrm{Ca}, \mathrm{Mg}$ e $\mathrm{S}$ foram de 27,$9 ; 4,3 ; 50,0 ; 15,0 ; 4,2$ e $2,1 \mathrm{~g} \mathrm{~kg}^{-1}$, respectivamente. Para o $\mathrm{B}, \mathrm{Cu}, \mathrm{Fe}, \mathrm{Mn}$ e $\mathrm{Zn}$, as concentrações médias estiveram em torno de 60,$5 ; 36 ; 284 ; 36$ e 121 $\mathrm{mg} \mathrm{kg}^{-1}$. Resultados semelhantes foram obtidos por Salinas et al. (1982), que não encontraram diferenças significativas entre os teores foliares de $\mathrm{N}, \mathrm{P}, \mathrm{K}, \mathrm{Ca}$, $\mathrm{Mg}, \mathrm{Fe}, \mathrm{Mn}, \mathrm{Cu}$ e $\mathrm{Zn}$, no período do florescimento, com o aumento da adição de B em solução nutritiva. Entretanto, esses autores obtiveram aumento na concentração de B nas folhas com o aumento do conteúdo desse elemento no substrato, o que também foi verificado por Santos et al. (1990), discordando, portanto, dos resultados obtidos nesse experimento. Com exceção do $\mathrm{Cu}$ e do
$\mathrm{Zn}$, os teores dos demais nutrientes apresentados estão dentro das faixas consideradas adequadas, segundo Trani \& Raij (1996). Os teores de $\mathrm{Cu}$ e de $\mathrm{Zn}$, por sua vez, estão acima dos limites máximos, possivelmente em virtude de pulverizações foliares feitas com fungicidas cúpricos (oxicloreto de cobre $500 \mathrm{~g} \mathrm{~kg}$ 1) e à base de zineb $\left(750 \mathrm{~g} \mathrm{~kg}^{-1}\right)$.

As doses de B não afetaram a composição mineral dos frutos. Os teores de nutrientes não diferiram significativamente no pericarpo e nas sementes, exceto o de B que foi superior nas sementes, nas quatro doses utilizadas (Tabela 2). Resultados concordantes foram obtidos por Navrot \& Levin (1976), que constataram que a aplicação de $2 \mathrm{~kg}$ ha${ }^{1}$ de B no solo não alterou os teores de $\mathrm{N}, \mathrm{Cu}, \mathrm{Zn}$ e Fe nos frutos. Por outro lado, Santos et al. (1990) verificaram que a aplicação de $1 \mathrm{mg} \mathrm{L}^{-1}$ de B em solução nutritiva aumentou os teores de $\mathrm{Mg}, \mathrm{Cu}$ 
e B nos frutos. Considerando-se os teores médios dos nutrientes nas partes dos frutos, verificou-se que as concentrações dos mesmos, exceto de $\mathrm{Ca}$ e de $\mathrm{S}$, de modo geral, foram maiores nas sementes. Os maiores teores de macronutrientes encontrados nos frutos foram os de $\mathrm{K} \mathrm{e}$ os de $\mathrm{N}$, seguidos dos de $\mathrm{P}$ e de $\mathrm{Mg}$. Os teores de $\mathrm{Ca}$ e de $\mathrm{S}$ foram encontrados aproximadamente na mesma proporção. Tais resultados estão parcialmente de acordo com os obtidos por Miller et al. (1979), que verificaram que os elementos mais absorvidos foram o $\mathrm{K}$ e o N seguido do $\mathrm{P}, \mathrm{S}, \mathrm{Ca}$ e $\mathrm{Mg}$. Quanto aos micronutrientes, a absorção em ordem decrescente foi: $\mathrm{Fe}, \mathrm{Mn}, \mathrm{Zn}, \mathrm{B}$ e Cu . Oliveira et al. (1971) verificaram, de maneira geral, resultados semelhantes.

Pode-se concluir pelos resultados obtidos, que em solo com 1,0 mg kg-1 de B a aplicação desse elemento via solo se mostrou desnecessária, o que sugere a necessidade de maiores estudos envolvendo diferentes tipos de solos e teores do elemento em questão, para que se possa recomendar quantidades mais adequadas de B para a cultura do pimentão.

\section{LITERATURA CITADA}

ADRIANO, D.C. Boron. In: ADRIANO, D.C. ed. Trace elements in the terrestrial environment. New York: Springer Verlag, 1986. p. 73-105.
BATAGLIA, O.C.; FURLANI, A.M.C.; TEIXEIRA, J.P.F.; FURLANI, P.R.; GALLO, J.R Métodos de análise química de plantas. Campinas: IAC, 1983. 48 p. (Boletim Técnico, 78).

BENINCASA, M.M.P. Análise de crescimento de plantas. Jaboticabal: FUNEP, 1988. $42 \mathrm{p}$. CORREIA, L.G.; CAMARGO, J.M.C.; SOUZA, E.L.S.; MONNERAT, P.H. Deficiência de magnésio e boro na fase inicial de crescimento do pimentão. In: CONGRESSO BRASILEIRO DE OLERICULTURA, 17., 1977, Juazeiro. Resumos... Juazeiro: EMBRAPA-CPATSA, 1977. p. 141-143.

GUNES, A. ALPASLAN, M.; CIKILI, Y.; OZCAN, H. Effect of zinc on the alleviation of boron toxicity in tomato. Journal of Plant Nutrition, v. 22, n. 7, p. 1061-1068, 1999.

HORINO, Y.; PINTO, C.M.F.; MAKISHIMA, N.; CORDEIRO, C.M.T. Efeito do boro e do zinco na produção de pimentão, Capsicum annuum L. In: CONGRESSO BRASILEIRO DE OLERICULTURA, 20., 1980, Brasília. Resumos... Brasília: EMBRAPA/EMBRATER/SOB, 1980. p. 99.

MILLER, C.H.; MCCOLLUM, R.E.; CLAIMON, S. Relationships between growth of bell peppers and nutrient accumulation during ontogeny in field environments. Journal of the American Society for Horticultural Science, St. Joseph, v. 104, n. 6, p. 852- 857, 1979.

NAVROT, J.; LEVIN, I. Effect of micronutrients on pepper (Capsicum annuum) grown in peat soil under greenhouse and field conditions. Experimental Agriculture, v. 12, n. 2, p. 129-133, 1976.

OLIVEIRA, G.D.; FERNANDES, P.D.; COSTA, M.C.B.; SANTOS, M.A.C.; HAAG, H.P. Nutrição mineral de hortaliças. XVI extração de micronutrientes por algumas hortaliças. O Solo, Piracicaba, v. 63, p. 11-14, 1971.
PASSOS, F.A. In: INSTITUTO AGRONÔMICO DE CAMPINAS. Recomendações de adubação e calagem para o Estado de São Paulo. Campinas: IAC, 1985. p.63. (Boletim Técnico, 100).

PANDEV, S.; STANEV, V.; K'DREV, T. Effect of deficiency in the minor elements $\mathrm{Fe}, \mathrm{Mn}, \mathrm{Cu}$, $\mathrm{Zn}$, Mo and B on some photosynthetic parameters of Capsicum. Fisiologiya na Rasteniyata, v. 7, n. 1, p. 46-54, 1981. Apud Horticultural Abstracts, Wallingford, v. 52, n. 1, p. 24. 1982. (Resumo). RAIJ, B. van; CANTARELLA, H.; QUAGGIO, J.A.; FURLANI, A.M.C. Recomendações de adubação e calagem para o Estado de São Paulo. 2ed. Campinas: IAC, 1996. 285 p. (Boletim Técnico, 100).

PLESE, L.P.M.; TIRITAN, C.S.; YASSUDA, E.I.; PROCHNOW, L.I.; CORRENTE, J.E.; MELLO, S.C. Efeitos das aplicações de cálcio e de boro na ocorrência de podridão apical e produção de tomate em estufa. Scientia Agrícola, Piracicaba, v. 55, n. 1, p. 144-148, 1998.

SALINAS, R.M.; CERDA, A.A.; FERNANDEZ, F.G. Efecto del boro sobre la composicion mineral de guisante y pimiento. Anales de Edafologia y Agrobiologia, v. 41, p. 991-1001, 1982.

SANTOS, I.S.; BARBEDO, C.J.; PIZIGATTI, R.; FERREIRA, J.M.; NAKAGAWA, J. Estudo da relação $\mathrm{Ca} \times \mathrm{B}$ na cultura do pimentão. Horticultura Brasileira, Brasília, v. 8, n. 2, p. 1923, 1990.

SARRUGE, J.R.; HAAG, H.P. Análises químicas em plantas. Piracicaba: ESALQ, Departamento de Química, 1974. 56 p.

TRANI, P.E.; RAIJ, B. van. Hortaliças. In: INSTITUTO AGRONÔMICO DE CAMPINAS. Recomendações de adubação e calagem para o Estado de São Paulo. Campinas: IAC, 1996. p. $155-$ 203. (Boletim Técnico, 100). 